\title{
ACTITUDES HACIA LAS RELACIONES DE PAREJA EN PERSONAS CON DISCAPACIDAD: ANÁLISIS DIFERENCIAL ENTRE FAMILIARES, PROFESIONALES Y POBLACION GENERAL
}

\author{
Gil-Juliá, B. \\ Dpto. de Personalidad, Evaluación y Tratamientos Psicológicos \\ Universitat de València, Valencia, España \\ Beatriz.Gil@uv.es \\ Gil-Llario, M.D. \\ Dpto. de Psicología Evolutiva y de la Educación \\ Universitat de València, Valencia, España \\ Fernández-García, 0. \\ Dpto. de Psicología Evolutiva y de la Educación \\ Universitat de València, Valencia, España \\ Nicola, Y. \\ Dpto. de Psicología Evolutiva y de la Educación \\ Universitat de València, Valencia, España
}

Esta investigación ha sido realizada en el marco del proyecto de investigación "Desarrollo y análisis de la eficacia de un programa de educación afectivo-sexual para prevenir el abuso y mejorar la salud sexual en personas con diversidad funcional intelectual" financiado por el Programa Estatal de l+D+i Orientada a los Retos de la Sociedad del Ministerio de Ciencia e Innovación (Proyecto RTI2018-095538-B-100).

\section{RESUMEN}

Las actitudes hacia la sexualidad de las personas con discapacidad parecen haber evolucionado en positivo. No obstante, todavía existen creencias erróneas que ayudan a mantener una actitud negativa hacia la sexualidad de estas personas dificultando su desarrollo psicosexual. Este trabajo analiza las actitudes hacia las relaciones de pareja de personas con discapacidad que poseen sus familiares, profesionales y población general. En el estudio participaron 252 personas: 86 familiares, 80 profesionales y 86 personas de la población general. Los resultados muestran que en todos los casos los familiares presentan menor tolerancia hacia las relaciones de pareja mientras que los profesionales han sido el grupo más tolerante. Concretamente, se observan diferencias estadísticamente significativas entre los tres grupos en la actitud hacia las relaciones de pareja ya sea entre personas con discapacidad intelectual ( $M=33$; $D T=3,12$ en familiares, $M=71,09$; $D T=5,13$ en profesionales y $M=63,14$; $D T=20,87$ en población general), sensorial ( $M=32,48 ; D T=3,49$ en familiares, $M=73,3 ; D T=4,06$ en profesionales y $M=70,79 ; D T=17,79$ en población general) o física ( $M=34,32 ; D T=3,02$ en familiares, $M=80,82 ; D T=4,82$ en 
profesionales y $M=66,60 ; D T=21,15$ en población general). Asimismo, los familiares muestran actitudes más desfavorables cuando las relaciones se establecen tanto entre parejas de distinto género $(F=275,08 ; p=, 000)$ como del mismo ( $F=319,92 ; p=, 000)$, así como entre parejas de igual edad $(F=424,79 ; p=, 000) 0$ diferente $(F=273,1 ; p=, 000)$. Estos datos remarcan la importancia de incluir módulos actitudinales en programas de educación afectivo- sexual dirigidos a los familiares puesto que permitiría no sólo mejorar la actitud de los padres sino también la que han podido aprender sus hijos favoreciendo así una adecuada expresión y desarrollo de su sexualidad.

Palabras clave: actitudes; discapacidad; relaciones de pareja; familiares; profesionales; población general

\begin{abstract}
Attitudes towards partner relationships in people with disabilities: differential analysis among family members, professionals and the general population. Attitudes toward the sexuality of people with disabilities seem to have evolved positively. However, there are still misconceptions that help to maintain a negative attitude toward the sexuality of these individuals, hindering their psychosexual development. This work analyzes the attitudes towards partner relationships of people with disabilities by their relatives, professionals, and the general population. 252 people participated in the study: 86 family members, 80 professionals and 86 people from the general population. The results show that in all cases family members have shown less tolerance towards partner relationships, while professionals were the most tolerant. Specifically, statistically significant differences are observed among the three groups in the attitude towards intimate relationships of people with intellectual disabilities $(M=33 ; S D=3.12$ in family members, $M=71.09 ; S D=5.13$ in professionals and $M=63.14$; $S D=20.87$ in the general population), sensory disabilities $(M=32.48 ; S D=3.49$ in family members, $M=73.3$; $S D=4.06$ in professionals and $M=70.79 ; S D=17.79$ in the general population) or physical disabilities ( $M=34.32$; $S D=3.02$ in family members, $M=80.82 ; S D=4.82$ in professionals and $M=66.60 ; S D=21.15$ in general population). Likewise, family members show more unfavorable attitudes when relationships are established both between different sex partners ( $F=275.08 ; p=.000$ ) and same sex partners ( $F=319.92 ; p=.000$ ), as well as between partners of the same age $(F=424.79 ; p=.000)$ or of different ages ( $F=273.1 ; p=.000)$. These data emphasize the importance of including attitudinal modules in affective-sexual education programs aimed at family. In this regard, we could not only improve their attitudes but also those attitudes that their children may learn from them, and thus favor an adequate expression and development of sexuality among people with disabilities.
\end{abstract}

Keywords: attitudes; disability; partner relationships; family; professionals; general population

\title{
ANTECEDENTES
}

La sexualidad es un aspecto fundamental de la experiencia humana (Evans, McGuire, Healy, \& Carley, 2009), presente a lo largo del ciclo vital que incluiría el sexo, la orientación sexual, la identidad, los papeles de género, el erotismo, el placer, la intimidad y la reproducción (Organización Panamericana de la Salud, 2018). Considerar de forma amplia y comprehensiva la sexualidad es necesario para poder entender mejor el concepto de salud sexual. Desde este marco se pretende promocionar resultados saludables de la sexualidad (p.e. auto-respeto, respeto mutuo, no explotación, satisfacción sexual, etc.) y evitar los negativos (p.e. embarazo no deseado, transmisión sexual de enfermedades, etc.) (Wilson, Parmenter, Stancliffe, \& Shuttleworth, 2011).

La salud sexual requiere de un abordaje respetuoso de la sexualidad y las relaciones sexuales y afectivas, sin discriminación ni violencia alguna. Por tanto, tal como contempla la Organización Mundial de la Salud y la Organización Panamericana de la Salud (2018), la salud sexual requiere de un enfoque positivo que garantice los derechos sexuales de todas las personas. No obstante, estos derechos a priori básicos no parecen estar tan claros cuando nos referimos a un sector particular de la sociedad como pueden ser las personas con algún grado de discapacidad. 
Si bien es cierto que las personas con discapacidad presentan un desarrollo sexual similar al del conjunto de la población, experimentando los mismos deseos y necesidades sexuales y de intimidad (Borawska-Charko, Rohleder, \& Finlay, 2017; Gil-Llario, Morell-Mengual, Ballester-Arnal, \& Díaz-Rodríguez, 2018; Rushbrooke, Murray, \& Townsend, 2014), también lo es que su sexualidad suele estar marcada por información inexacta y engañosa llegando a experimentar dificultades en este ámbito. Algunas de ellas, podrían estar relacionadas con el comportamiento que llevan a cabo o la expresión de sentimientos debido a baja autoeficacia (Eastgate, 2008; Rushbrooke et al., 2014), pobres habilidades de negociación sexual (Dawood, Bhagwanjee, Govender, \& Chohan, 2006), e incluso dificultad para aprender comportamientos sexuales "socialmente aceptables" (Kramers-Olen, 2016). En cambio, otras dificultades que podemos encontrar en la expresión de su sexualidad podrían no estar relacionadas con la discapacidad sino más bien con otras variables como la falta de educación sexual (García \& de Dios, 2014; Gil, Díaz, Ceccato, Ballester, \& Giménez, 2014), la sobreprotección familiar, el no reconocimiento de su necesidad de intimidad sexual (de Dios, García, \& Suárez, 2006) y la reducción de sus principales interacciones a familiares/profesionales (Navarro, Torrico, \& López, 2010), privándoles de la interacción con iguales.

No cabe duda de que la sexualidad de las personas con discapacidad está influida por mitos y falsas creencias. Numerosas investigaciones reflejan entre los principales mitos en torno a la sexualidad de las personas con discapacidad los siguientes: no tienen sexualidad, son "niños eternos", no están interesadas en su propia sexualidad 0 la de los demás, son personas promiscuas o "hipersexuales" (Eastgate, 2008; Wilkinson, Theodore, \& Raczka, 2015).

Todos estos mitos, aun sin poseer base científica, pueden influir en las actitudes que se muestran hacia estas personas y su sexualidad actuando como importantes barreras de un adecuado desarrollo psicosexual (Navarro et al., 2010). Si bien estudios recientes parecen sugerir actitudes algo más liberales sobre la sexualidad de las personas con discapacidad, las oportunidades para expresar de forma libre y adecuada su sexualidad siguen siendo limitadas y controladas por otros (Eastgate, 2008; Wilkinson et al., 2015).

Diversos estudios sobre las actitudes hacia la sexualidad de las personas con discapacidad muestran resultados positivos cuando se trata de aspectos relacionados con el derecho a una vida emocional/sexual plena, recibir educación sexual o realizar manifestaciones de afecto (besos, caricias 0 abrazos) (Cuskelly \& Bryde, 2004; Gilmore \& Chambers, 2010). No obstante, las actitudes tienden a ser más conservadoras al abordar otros aspectos como el matrimonio/relaciones de pareja, paternidad/maternidad (Hasson-Ohayon, Hertz, Vilchinsky, \& Kravetz, 2014; Morell-Mengual, Gil-Llario, Díaz-Rodríguez, \& Caballero-Gascón, 2017; Olavarrieta et al., 2013). Asimismo, algunas investigaciones sobre las actitudes hacia la sexualidad de las personas con discapacidad indican que estas actitudes pueden variar en función del grupo poblacional analizado. En este sentido, varios estudios indican que los familiares más directos tienden a presentar actitudes más negativas hacia la sexualidad de las personas con discapacidad (Bazzo, Nota, Soresi, Ferrari, \& Minnes, 2007; Cuskelly \& Bryde, 2004; Olavarrieta et al., 2013), frente a los profesionales (Evans et al., 2009; Gilmore \& Chambers, 2010) y población general (Cuskelly \& Bryde, 2004; Caballero-Gascón, Gil-Llario, Morell-Mengual, Fernández-García, \& Gil-Juliá, 2017; Díaz-Rodríguez, Gil-Llario, Morell-Mengual, Salmerón-Sánchez, \& Ruiz-Palomino, 2016).

Si bien las actitudes hacia la sexualidad de las personas con discapacidad parecen haber evolucionado en positivo a lo largo de los años, todavía existen creencias erróneas que ayudan a mantener una actitud negativa dificultando su desarrollo psicosexual. Este trabajo pretende explorar las actitudes hacia las relaciones de pareja de personas con discapacidad que poseen sus familiares, profesionales y población general. Se tuvieron en cuenta variables como el tipo de discapacidad (intelectual, sensorial y física), quién presentaba la discapacidad (ambos miembros de la pareja/sólo uno de ellos), la orientación sexual de las parejas y la edad. 


\section{MÉTODO}

\section{Participantes}

En el estudio participaron 252 personas procedentes de tres grupos: 86 familiares, 80 profesionales y 86 personas de la población general. En los tres grupos la distribución de la muestra según el sexo ha sido muy homogénea. El grupo de familiares está formado por 43 hombres y 43 mujeres; el de profesionales por 39 hombres y 41 mujeres; y el de la población general lo componen 43 hombres y 43 mujeres. En los tres grupos la edad ha oscilado entre 36 y 82 años (Tabla 1).

Tabla 1. Características sociodemográficas

\begin{tabular}{lllll}
\hline \multirow{3}{*}{ Género } & & Familiares & Profesionales & $\begin{array}{l}\text { Población } \\
\text { general }\end{array}$ \\
& Hombre & $50 \%$ & $48,8 \%$ & $50 \%$ \\
\multirow{3}{*}{ Edad } & Mujer & $50 \%$ & $51,2 \%$ & $50 \%$ \\
& $36-51$ años & $4,7 \%$ & $71,3 \%$ & $47,7 \%$ \\
\multirow{5}{*}{ Nivel formación } & 52-66 años & $51,2 \%$ & $27,5 \%$ & $47,7 \%$ \\
& 67-82 años & $44,2 \%$ & $1,3 \%$ & $4,7 \%$ \\
& Sin estudios & $12,8 \%$ & $0 \%$ & $4,7 \%$ \\
& Graduado escolar & $55,8 \%$ & $1,3 \%$ & $32,6 \%$ \\
& Educación secundaria & $22,1 \%$ & $60 \%$ & $33,7 \%$ \\
& Educación superior & $9,3 \%$ & $38,8 \%$ & $29,1 \%$ \\
\hline
\end{tabular}

\section{Instrumentos}

En este estudio inicialmente se utilizó un cuestionario Ad-Hoc de información sociodemográfica que incluye la edad, el género y el nivel de formación. Asimismo, todos los participantes cumplimentaron la Escala de Actitudes hacia las Relaciones Sexuales de las personas con Discapacidad Intelectual (ARSEXDI) (Gil-Llario \& Díaz-Rodríguez, 2017). Consta de 17 ítems donde se evalúan las actitudes hacia combinaciones de parejas que resultan del cruce entre 4 variables: el género de los miembros de la pareja (mismo vs distinto género), su edad (misma vs distinta edad), el tipo de discapacidad (intelectual, sensorial o física) y quién presenta la discapacidad (ambos o sólo uno de ellos).

\section{Procedimiento}

Para recoger la muestra de la población general se contactó, vía correo electrónico, con diversas asociaciones españolas ofreciéndoles información sobre el estudio que nos disponíamos a desarrollar y solicitándoles su colaboración. Estas asociaciones divulgaron, a través de sus páginas web y/o sus redes sociales, un texto que contenía una breve explicación del estudio y un enlace que remitía a los cuestionarios online. Cuando el participante accedía a la encuesta online se le proporcionaba información más detallada sobre los objetivos del estudio y el tratamiento estadístico de los datos, y se obtenía su consentimiento informado.

Para recoger la muestra de profesionales y familiares de personas con discapacidad intelectual (DI) se contactó con diversos directores/as de distintos centros ocupacionales proporcionándoles información sobre el estudio. Los centros se encargaron de difundir los cuestionarios entre los profesionales y familiares para su cumplimentación. 


\section{Análisis de datos}

Los datos se analizaron mediante el paquete estadístico SPSS Versión 26.0. Se realizaron análisis descriptivos para explorar las actitudes de familiares, profesionales y población general hacia las relaciones de pareja de personas con DI, sensorial (DS) y física (DF). Para analizar las posibles diferencias entre los tres grupos en las actitudes que presentan hacia las relaciones de pareja se llevaron a cabo análisis ANOVA de un factor.

\section{RESULTADOS}

El análisis de las actitudes hacia las relaciones de pareja muestra diferencias estadísticamente significativas entre los tres grupos tanto en las relaciones entre personas con DI ( $F=212,02 ; p=, 000)$, como DS ( $F=377,51$; $p=, 000)$ o DF $(F=291,37 ; p=, 000)$ siendo en todos los casos los familiares quienes presentan menor tolerancia hacia las relaciones de pareja mientras que los profesionales han sido el grupo más tolerante (Figura 1). Las pruebas post hoc mediante Bonferroni determinan diferencias significativas entre familiares y profesionales, así como entre familiares y población general en los tres tipos de discapacidad ( $p=, 000$ en los tres tipos). Asimismo, las diferencias también han sido significativas entre profesionales y población general en DI $(p=, 000)$ y DF $(p=, 000)$ pero no en DS $(p=, 412)$ (Figura 1$)$.

Figura 1. Actitudes hacia las relaciones de pareja de personas con distinto tipo de discapacidad

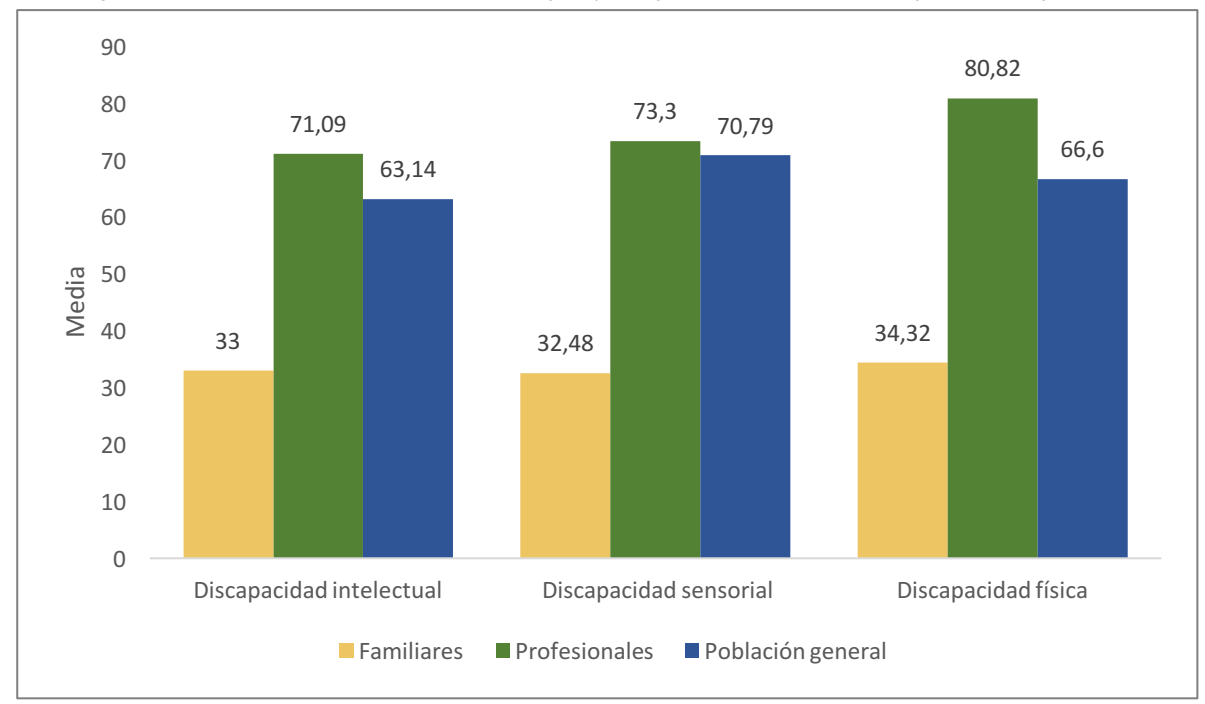

De igual forma los familiares han mostrado actitudes más desfavorables que los otros grupos tanto cuando las relaciones se establecen entre parejas formadas por dos personas con discapacidad, como cuando la presenta un solo miembro, con independencia del tipo de discapacidad (Tabla 2). 
Tabla 2. Medias en actitud hacia las relaciones de pareja en función de que la discapacidad la presenten los dos o un solo miembro de la pareja

\begin{tabular}{|c|c|c|c|c|c|}
\hline & $\begin{array}{l}\text { Familiares }^{1} \\
\text { M (DT) }\end{array}$ & $\begin{array}{l}\text { Profesionales }^{2} \\
\text { M (DT) }\end{array}$ & $\begin{array}{l}\text { Población } \\
\text { general }^{3} \\
\text { M (DT) }\end{array}$ & $\mathrm{F}$ & Bonferroni \\
\hline RS 2 DI & $\begin{array}{l}17,31 \\
(2.38)\end{array}$ & $32,69(2,70)$ & $26,45(8,94)$ & $157,66 * * *$ & $\begin{array}{l}(1<2) \\
(2>3)\end{array}$ \\
\hline RS 2 DS & $\begin{array}{l}16,93 \\
(2,68)\end{array}$ & $33,90(2,04)$ & $29,21(7,21)$ & $299,52 * * *$ & $\begin{array}{l}(1<2) \\
(2>3)\end{array}$ \\
\hline RS 2 DF & $\begin{array}{l}18,42 \\
(2,64)\end{array}$ & $33,94(2,18)$ & $26,95(8,73)$ & $167,92 * * *$ & $\begin{array}{l}(1<2) \quad(1<3) \\
(2>3)\end{array}$ \\
\hline RS 1 DI & $\begin{array}{l}15,69 \\
(1,87)\end{array}$ & $38,40(3,16)$ & $36,69(13,53)$ & $202,82 * * *$ & $(1<2)(1<3)$ \\
\hline RS $1 \mathrm{DS}$ & $\begin{array}{l}15,55 \\
(1,86)\end{array}$ & $39,4(2,65)$ & $41,58(11,47)$ & $367,76^{* * *}$ & $(1<2)(1<3)$ \\
\hline RS $1 \mathrm{DF}$ & $\begin{array}{l}15,91 \\
(1,86)\end{array}$ & $46,89(2,93)$ & $39,65(13,08)$ & $354,30 * * *$ & $\begin{array}{l}(1<2) \\
(2>3)\end{array}$ \\
\hline
\end{tabular}

$* * * \mathrm{p}<, 001$

Nota: Actitudes hacia las relaciones entre: RS 2 DI: ambos con DI; RS 2 DS: ambos con DS; RS 2 DF: ambos con DF; RS 1 DI: solo uno con DI; RS 1 DS: solo uno con $D S$; $R S 1 D F$ : solo uno con DF.

Analizando el grado de aceptación hacia las parejas formadas por personas con discapacidad con el mismo 0 diferente género, los resultados en general muestran diferencias significativas tanto en parejas de género distinto ( $F=275,08 ; p=, 000$ ) como del mismo género ( $F=319,92 ; p=, 000)$. En el caso de parejas del mismo género, las diferencias entre los tres grupos se observan tanto en parejas de chicos $(F=331,36 ; p=, 000)$ como en parejas de chicas ( $F=377,69 ; p=, 000)$. Estas diferencias indican nuevamente una actitud más desfavorable por parte de Ios familiares y más favorable en los profesionales (Tabla 3). Profundizando en este análisis mediante pruebas post hoc observamos diferencias significativas entre familiares y profesionales, así como entre familiares y población general tanto en parejas de distinto como del mismo género ( $p=, 000$ en los dos casos). Asimismo, las diferencias también han sido significativas entre profesionales y población general en parejas del mismo género $(p=, 000)$ pero no en parejas de distinto género $(p=, 141)$. Esta actitud más desfavorable de los familiares también se observa cuando las relaciones se establecen tanto entre parejas de distinto género como del mismo género con independencia de la discapacidad (Tabla 3). 
PSICOLOGÍA Y CRECIMIENTO HUMANO

Tabla 3. Medias en actitud hacia las relaciones de pareja formadas por personas del mismo o distinto género

\begin{tabular}{|c|c|c|c|c|c|}
\hline & $\begin{array}{l}\text { Familiares }^{1} \\
\mathrm{M}(\mathrm{DT})\end{array}$ & $\begin{array}{l}\text { Profesionales }^{2} \\
\text { M (DT) }\end{array}$ & $\begin{array}{l}\text { Población } \\
\text { general }^{3} \\
\text { M (DT) }\end{array}$ & $\mathrm{F}$ & Bonferroni \\
\hline RS homo & $\begin{array}{l}35,81 \\
(3,14)\end{array}$ & $85,29(8,97)$ & $70,07(20,19)$ & $319,92 * * *$ & $\begin{array}{ll}(1<2) & (1<3) \\
(2>3) & \end{array}$ \\
\hline RS hetero & $\begin{array}{l}56,10 \\
(3,98)\end{array}$ & $111,70(3,97)$ & $\begin{array}{l}106,42 \\
(28,61)\end{array}$ & $275,08 * * *$ & $(1<2)(1<3)$ \\
\hline RS.homoDI & $\begin{array}{l}11,61 \\
(1,79)\end{array}$ & $28,44(2,98)$ & $22,26(7,67)$ & $251,54 * * *$ & $\begin{array}{l}(1<2) \\
(2>3)\end{array} \quad(1<3)$ \\
\hline RS.homoDS & $\begin{array}{l}11,67 \\
(1,74)\end{array}$ & $28,50(2,86)$ & $24,73(6,59)$ & $354,99 * * *$ & $\begin{array}{ll}(1<2) & (1<3) \\
(2>3) & \end{array}$ \\
\hline RS.homoDF & $\begin{array}{l}12,52 \\
(1,68)\end{array}$ & $28,35(3,28)$ & $23,08(7,72)$ & $220,01 * * *$ & $\begin{array}{l}(1<2) \quad(1<3) \\
(2>3)\end{array}$ \\
\hline RS.heteroDI & $\begin{array}{l}18,76 \\
(2,35)\end{array}$ & $33,26(2,75)$ & $33,38(11,08)$ & $130,17 * * *$ & $(1<2)(1<3)$ \\
\hline RS.heteroDS & $\begin{array}{l}18,17 \\
(2,45)\end{array}$ & $35,39(2,31)$ & $37,59(9,38)$ & $285,61 * * *$ & $\begin{array}{l}(1<2) \quad(1<3) \\
(2>3)\end{array}$ \\
\hline RS.heteroDF & $\begin{array}{l}19,17 \\
(2,27)\end{array}$ & $43,05(1,59)$ & $35,44(11,14)$ & $277,46 * * *$ & $\begin{array}{ll}(1<2) & (1<3) \\
(2>3) & \\
\end{array}$ \\
\hline
\end{tabular}

Nota: Actitudes hacia las relaciones entre: RS homo: ambos mismo género; RS hetero: distinto género; RS.homoDI: ambos mismo género, con DI; RS.homoDS: ambos mismo género, con DS; RS.homoDF: ambos mismo género, con DF; RS.heteroDI: distinto género, con DI; RS.heteroDS: distinto género, con DS; RS.heteroDF: distinto género, con $D F$.

Por lo que respecta a la edad, de manera global los resultados también revelan diferencias significativas entre Ios tres grupos tanto entre parejas de edades cercanas $(F=424,79 ; p=, 000)$ como con mucha diferencia de edad entre ellos ( $F=273,1 ; p=, 000)$. Al igual que en las situaciones anteriores se observa una actitud menos tolerante por parte de la familia y más tolerante por los profesionales, con independencia de que los miembros de la pareja con discapacidad tengan o no la misma edad (Tabla 4). Las pruebas post hoc mediante Bonferroni determinan diferencias significativas entre familiares y profesionales, familiares y población general, así como entre profesionales y población general tanto en parejas de la misma edad como de edades diferentes ( $p \leq, 001$ en todas las comparaciones). Esta actitud menos tolerante de los familiares podemos observarla igualmente en los distintos tipos de discapacidad cuando las relaciones se establecen entre parejas de la misma edad y cuando los dos miembros de la pareja presentan diferente edad (Tabla 4). Además, si profundizamos un poco más en el análisis de las parejas de distinta edad, las diferencias entre los tres grupos han aparecido y con la misma tendencia que en las situaciones anteriores (es decir, peor tolerancia en familiares y mejor tolerancia en profesionales), con independencia de que fuera mayor el chico $(F=172,35 ; p=, 000)$ o la chica $(F=225,34 ; p=, 000)$. 
Tabla 4. Medias en actitud hacia las relaciones de pareja formadas por personas de igual o distinta edad

\begin{tabular}{|c|c|c|c|c|c|}
\hline & $\begin{array}{l}\text { Familiares }^{1} \\
\text { M (DT) }\end{array}$ & $\begin{array}{l}\text { Profesionales }^{2} \\
\text { M (DT) }\end{array}$ & $\begin{array}{l}\text { Población } \\
\text { general }^{3} \\
\text { M (DT) }\end{array}$ & $\mathrm{F}$ & Bonferroni \\
\hline RS. misma edad & $\begin{array}{l}33,88 \\
(4,15)\end{array}$ & $92,07(7,15)$ & $\begin{array}{l}83,88 \\
(22,65)\end{array}$ & $424,79 * * *$ & $\begin{array}{l}(1<2) \\
(1<3) \\
(2>3)\end{array}$ \\
\hline RS. distinta edad & $\begin{array}{l}65,92 \\
(4,33)\end{array}$ & $133,14(6,35)$ & $\begin{array}{l}116,65 \\
(32,41)\end{array}$ & $273,10 * * *$ & $\begin{array}{l}(1<2) \\
(1<3) \\
(2>3)\end{array}$ \\
\hline RS.misma.E.DI & $\begin{array}{l}11,40 \\
(1,54)\end{array}$ & $28,82(2,91)$ & $\begin{array}{l}26,52 \\
(8,67)\end{array}$ & $259,25 * * *$ & $\begin{array}{l}(1<2) \\
(1<3) \\
(2>3)\end{array}$ \\
\hline RS.misma.E.DS & $\begin{array}{l}11,33 \\
(1,99)\end{array}$ & $30,36(2,46)$ & $\begin{array}{l}29,59 \\
(7,40)\end{array}$ & $448,47 * * *$ & $\begin{array}{l}(1<2) \\
(1<3)\end{array}$ \\
\hline RS.misma.E.DF & $\begin{array}{l}11,15 \\
(1,72)\end{array}$ & $32,88(2,55)$ & $\begin{array}{l}27,77 \\
(8,84)\end{array}$ & $364,78 * * *$ & $\begin{array}{l}(1<2) \\
(1<3) \\
(2>3)\end{array}$ \\
\hline RS.diferente.E.DI & $\begin{array}{l}21,59 \\
(2,63)\end{array}$ & $42,26(2,63)$ & $\begin{array}{l}36,62 \\
(12,47)\end{array}$ & $166,28 * * *$ & $\begin{array}{l}(1<2) \\
(1<3) \\
(2>3)\end{array}$ \\
\hline RS.diferente.E.DS & $\begin{array}{l}21,15 \\
(2,39)\end{array}$ & $42,94(2,19)$ & $\begin{array}{l}41,19 \\
(10,67)\end{array}$ & $292,99 * * *$ & $\begin{array}{l}(1<2) \\
(1<3)\end{array}$ \\
\hline $\begin{array}{l}\text { RS. } \\
\text { diferente.E.DF }\end{array}$ & $\begin{array}{l}23,17 \\
(2,61)\end{array}$ & $47,94(2,55)$ & $\begin{array}{l}38,84 \\
(12,49)\end{array}$ & $227,04 * * *$ & $\begin{array}{l}(1<2) \\
(1<3) \\
(2>3)\end{array}$ \\
\hline
\end{tabular}

$* * * \mathrm{p}<, 001$

Nota: Actitudes hacia las relaciones entre: RS. misma edad: ambos misma edad; RS. distinta edad: distinta edad; RS.misma.E.DI: ambos misma edad, con DI; RS.misma.E.DS: ambos misma edad, con DS; RS.misma.E.DF: ambos misma edad, con DF; RS.distinta.E.DI: distinta edad, con DI; RS.distinta.E.DS: distinta edad, con DS; RS.distinta.E.DF: distinta edad, con DF.

\section{DISCUSIÓN Y CONCLUSIONES}

Los resultados de este trabajo nos permiten conocer con más detalle las actitudes que presentan familiares, profesionales y población general hacia las relaciones de pareja de personas con distinto tipo de discapacidad, al tener en cuenta variables poco exploradas hasta el momento relativas a las personas con discapacidad, como por ejemplo si la discapacidad la presentaban ambos miembros de la pareja o sólo uno de ellos.

En líneas generales la tolerancia mostrada por los tres grupos hacia las relaciones de pareja ha sido diferente tanto en la DI, como DS y DF, en todas las condiciones analizadas. En este sentido, nuestro estudio revela que son los familiares quienes presentan menor tolerancia hacia las relaciones de pareja mientras que los profesionales han sido el grupo más tolerante. Estos datos van en la línea de investigaciones previas que muestran actitudes hacia la sexualidad muy conservadoras en los familiares, mientras que los profesionales de la salud poseen 
actitudes mucho más liberales (Bazzo et al., 2007; Cuskelly \& Bryde, 2004; Evans et al., 2009; Gilmore \& Chambers, 2010; Olavarrieta et al., 2013). Asimismo, en cuanto al tipo de discapacidad, en principio nuestros datos serían congruentes en parte con la literatura revisada que ofrece diferencias en las actitudes frente a la DI y DF (Katz, Shemesh, \& Bizman, 2000; Morell-Mengual et al., 2017; Tsang, Chan, \& Chan, 2004) pues las puntuaciones obtenidas son ligeramente más positivas en la DF, sobre todo en los profesionales. No obstante, seguimos encontrando que la actitud de la familia es poco tolerante en los tres tipos de discapacidad con valores muy similares. Ello puede deberse a que en nuestro estudio estamos evaluando actitudes hacia las relaciones de pareja, aspecto que suele despertar actitudes más conservadoras según estudios previos (Hasson-Ohayon et al., 2014; Olavarrieta et al., 2013).

Otro aspecto considerado relevante en nuestro estudio ha sido valorar las actitudes hacia las relaciones de pareja en función de que los dos miembros presentaran discapacidad o sólo uno de ellos, habiendo obtenido en ambas circunstancias una actitud más desfavorable por parte de la familia, y además en los tres tipos de discapacidad. Estos hallazgos no hemos podido contrastarlos al no haber encontrado estudios previos que incluyeran esta variable.

Respecto al género de los miembros de la pareja, nuestros datos muestran de nuevo peor tolerancia por parte de la familia tanto cuando las relaciones de pareja se dan entre personas de igual o distinto género, con independencia de que las parejas estén formadas por dos chicos o dos chicas. Estos datos irían en parte en la misma dirección que los encontrados en estudios previos (Caballero-Gascón et al., 2017) que muestran que mantener relaciones con personas de su mismo 0 de distinto género no influye a la hora de modular la tolerancia hacia las relaciones sexuales en discapacidad.

Finalmente, en lo relativo a la edad de las personas con discapacidad, los resultados también revelan actitudes más desfavorables por parte de los familiares tanto en parejas de igual edad como de diferente, en contra de lo que cabría esperar inicialmente teniendo en cuenta la literatura que muestra actitudes más favorables cuando las edades son similares (Morales, López, \& Mullet, 2011). Ello reflejaría ese posible miedo a que la diferencia de edad pudiera llevar a situaciones de desigualdad dentro de la pareja y por tanto abuso (Esterle, Muñoz, \& Mullet, 2008) aspecto que no ha influido en la actitud de nuestros participantes.

En resumen, estos datos remarcan la importancia de seguir trabajando en la mejora de las actitudes hacia la sexualidad de las personas con discapacidad, y de manera particular las de los familiares, quienes han mostrado peor tolerancia. Incluir módulos actitudinales en programas de educación afectivo- sexual dirigidos a los familiares nos permitiría mejorar no sólo la actitud de los padres sino también la que han podido aprender sus hijos, al internalizar los mensajes recibidos, y así favorecer una adecuada expresión y desarrollo de su sexualidad.

\section{REFERENCIAS BIBLIOGRÁFICAS}

Bazzo, G., Nota, L., Soresi, S., Ferrari, L., \& Minnes, P. (2007). Attitudes of Social Service Providers towards the Sexuality of Individuals with Intellectual Disability. Journal of Applied Research in Intellectual Disabilities, 20, 110-115

Borawska-Charko, M., Rohleder, P., \& Finlay, W.M.L. (2017). The Sexual Health Knowledge of People with Intellectual Disabilities: a Review. Sexuality Research and Social Policy, 14, 393-409.

Caballero-Gascón, L., Gil-Llario, M.D., Morell-Mengual, V., Fernández-García, O., \& Gil-Juliá, B. (2017). Actitudes de la población general hacia las relaciones sexuales de las personas con diversidad funcional: variables moduladoras. International Journal of Developmental and Educational Psychology, 1(3), 103-110.

Cuskelly, M., \& Bryde, R. (2004). Attitudes towards the sexuality of adults with an intellectual disa- bility: parents, support staff, and a community sample. Journal of Intellectual and Developmental Disability, 29(3), 255-264. https://doi.org/10.1080/13668250412331285136

Dawood, N., Bhagwanjee, A., Govender, K., \& Chohan, E. (2006). Knowledge, attitudes and sexual practices of adolescents with mild retardation, in relation HIV/AIDS. African Journal of AIDS Research, 5(1), 1-10. 
De Dios, R., García, M., \& Suárez, 0. (2006). Discapacidad intelectual y sexualidad: Conductas sexuales socialmente no aceptadas. Serie documentos técnicos. Asturias: Gobierno del Principado de Asturias. Consejería de Vivienda y Bienestar Social.

Díaz-Rodríguez, I., Gil-Llario, M.D., Morell-Mengual, V., Salmerón-Sánchez, P., \& Ruiz-Palomino, E. (2016). Actitudes hacia la sexualidad: ¿Difieren las personas con discapacidad intelectual de la población general? International Journal of Developmental and Educational Psychology, 1(1), 235-242.

Eastgate, G. (2008). Sexual health for people with intellectual disability. Salud Publica de Mexico, 50 (Suppl. 2), s255-s259.

Esterle, M., Muñoz, M. T., \& Mullet, E. (2008). Judging the acceptability of sexual intercourse among people with learning disabilities: French laypeople's viewpoint. Sexuality and Disability, 26(4), 219-227.

Evans, D. S., McGuire, B. E., Healy, E., \& Carley, S. N. (2009). Sexuality and personal relationships for people with an intellectual disability. Part II: Staff and family carer perspectives. Journal of Intellectual Disability Research, 53, 913-921.

García, M. \& de Dios, R. (2014). Guía de educación sexual y discapacidad. Asturias: Gobierno del Principado de Asturias. Consejería de Vivienda y Bienestar Social

Gil, M. D., Díaz, I., Ceccato, R., Ballester. R., \& Giménez, C. (2014). Conocimientos, actitudes hacia la orientación sexual, comportamientos de riesgo y abusos sexuales en mujeres con discapacidad intelectual. International Journal of Developmental and Educational Psychology, 1(2), 397-406.

Gil-Llario, M.D. \& Díaz-Rodríguez, I. (2017). Construcción de la salud sexual y análisis de su influencia en la calidad de vida y prevención de abusos sexuales en personas adultas con discapacidad intelectual (Tesis doctoral). Universitat de València, Valencia (España).

Gil-Llario, M.D., Morell-Mengual, V., Ballester-Arnal, R., \& Díaz-Rodríguez, I. (2018). The Experience of sexuality in adults with intellectual disability. Journal of Intellectual Disability Research, 62(1), 72-80. https://doi.org/10.1111/jir.12455

Gilmore, L. \& Chambers, B. (2010). Intellectual disability and sexuality: attitudes of disability support staff and leisure industry employees. Journal of Intellectual and Developmental Disability, 35(1). pp. 1-22.

Hasson- Ohayon, I., Hertz,I., Vilchinsky, N. y Kravetz, S. (2014). Attitudes toward the sexuality of persons with physical versus psychiatric disabilities. Rehabilitation Psychology, 59(2). pp. 236-241.

Katz, S., Shemesh, T. y Bizman, A. (2000). Attitudes of university students towards the sexuality of persons with mental retardation and persons with paraplegia. British Journal Developmental Disabilities, 46, 109-117.

Kramers-Olen, A. (2016). Sexuality, intellectual disability, and human rights legislation. South African Journal of Psychology, 46(4), 504-516.

Morales, G.E., López, E.0., \& Mullet, E. (2011). Acceptability of Sexual Relationships Among People with Learning Disabilities: Family and Professional Caregivers' Views in Mexico. Sexuality \& Disability, 29, 165174.

Morell-Mengual, V., Gil-Llario, M.D., Díaz-Rodríguez, I., \&Caballero-Gascón, L. (2017). Actitudes de padres, profesionales y población general hacia la sexualidad de las personas con discapacidad física e intelectual. International Journal of Developmental and Educational Psychology , 1(3), 173-184.

Navarro, Y., Torrico, E., \& López, M.J. (2010). Programa de intervención psicosexual en personas con discapacidad intelectual. Educación y Diversidad, 4(2), 75-92.

Olavarrieta, S., Darín, L., Suárez, P., Tur, N., Besteiro, B., Gómez-Jarabo, G. (2013). Actitudes hacia la sexualidad, esterilización, maternidad/paternidad y habilidades de crianza de personas con discapacidad intelectual: un estudio preliminar. Siglo Cero, 44(4), 55-69.

Organización Panamericana de la Salud. (2018). Comunicaciones breves relacionadas con la sexualidad. Recomendaciones para un enfoque de salud pública. Washington, D.C.: Organización Panamericana de la Salud. https://iris.paho.org/bitstream/handle/10665.2/49504/9789275320174_spa.pdf?ua=1 
Rushbrooke, E., Murray, C., \& Townsend, S. (2014). The experiences of intimate relationships by people with intellectual disabilities: A qualitative study. Journal of Applied Research in Intellectual Disabilities, 27, 531541.

Tsang, H., Chan, F., y Chan, C. C. H. (2004) Attitudes of occupational therapy students toward placement of treatment facilities in the comunity: A conjoint analysis. American Journal of Occupational Therapy, 58, 426-434.

Wilkinson, V. J., Theodore, K., \& Raczka, R. (2015). 'As normal as possible': Sexual identity development in people with intellectual disabilities transitioning to adulthood. Sexuality and Disability, 33, 93-105.

Wilson, N. J., Parmenter, T. R., Stancliffe, R. J., \& Shuttleworth, R. P. (2011). Conditionally sexual: Men and teenage boys with moderate to profound intellectual disability. Sexuality and Disability, 29, 275-289. 
